\section{Respiratory Disturbance Index}

Helga Peter

Marburg, Deutschland

\section{Definition}

Anzahl der Apnoen/Hypopnoen und der Respiratory Effort Related Arousals (RERAs) pro Stunde Schlafzeit.

\title{
Synonyme
}

RDI 